\title{
Offering Library Resources through Web-site and Mobile Systems with Web Services for Central Library Suratthani Rajabhat University
}

\author{
Sarawut Markchit \\ Faculty of Science and Technology, Suratthani Rajabhat University, Thailand
}

Copyright (C) 2015 Horizon Research Publishing All rights reserved.

\begin{abstract}
The purposes of this research were firstly, to identify the problem and obtain necessary requirements data from the librarians. Secondly, to analyze the obtain data, design and develop the system. Thirdly, to conduct an assessment or survey from the users of library resources service through websites and mobile systems with web services with regards overall performance of Suratthani Rajabhat University Central Library. The researcher obtained the data from the users through questionnaires and directs interview, after the feedback was consolidated, the obtained information were analyzed, designed and developed the system. The instruments used for the development were visual studio .NET (ASP.NET) for web-based application, HTML5 for mobile application, web-service for data communication with the component and connected to staff system of this University, Microsoft SQL server for database management and longest matching algorithm for data retrieval. After installed the system, assess for the users satisfaction such as easily to use, method of output, prompt service, accuracy, security, completely, increase efficacy for work and benefit from the system showed that the total average as satisfactory.
\end{abstract}

Keyword Offering System, Library Resource, Mobile Application, Web-service

\section{Introduction}

The library is where assemble a lot of resources such as book, journal, many printed materials and other media. The library must be provide and manage the items to serve to the library users. The objectives of library are to provide education, knowledge, research and relaxing. The good library should provide the service based on the requirement from the users. The acquisition form is a vital link in the cycle of publishing, selection, request and providing materials for users. The imperatives acquisitions for the staff are to acquire materials as quickly and economically as possible, while offering an efficient and responsive service
[6]. The Library exists to serve the need of their users. This service philosophy has always been a basic tenet of our profession. In the management of information resources too, librarians must seek to serve the needs of their users. The difficulty is that, while some need of current users not even know of the existence of all that they need to have, or have access to. Hence, serving the need of users is not a passive but an active role [7].

The Central library of Suratthani Rajabhat University has many resources to service the library users because it's obligation of the university to provide quality and education support, research, art and culture maintain, academic service for teachers' students and other users. The library mission is to provide enough resources for the users. In the past the library disregards the quality service. Hence, the library permits users to offer the library resources through email, telephone or direct to the librarian. The consequence, the service is too slow, redundant, lost and wrong data.

Therefore, the Central Library of Suratthani Rajabhat University wants to offer library resources system through web-based application and mobile application to provide the library quality service such as increase the accuracy, prompt of service, reducing of the work of librarians and direct to user requirement. Last thing, users can offer the library resources ubiquitous access.

\section{Background}

A library is an organized collection of sources of information and similar resources, made accessible to a defined community for reference or borrowing [10]. It provides physical or digital access to material, and may be a physical building or room, or a virtual space, or both [2]. A library's collection can include book, periodicals, newspapers, manuscripts, films, maps, prints, document, microform, CDs, cassettes, videotapes, DVDs, Blu-ray Discs, e-book, databases and other formats. A library computer system is the software used to catalog, track circulation (where appropriate) and inventory a library's assets. It is 
intended for home, church, private enterprise or other small to medium sized collections. Larger libraries will typically use an integrated library system to manage the more complex activities such as acquisitions, inter library loan, and the licensing of online resources. Library automation is the general term for information and communications technologies (ICT) that are used to replace manual systems in the library. The functions that may be automated are any or all of the following: acquisition, cataloging, circulation, serials management and reference.

Library acquisitions are the department of a library responsible for the selection and purchase of materials or resources. The department may select vendors, negotiate consortium pricing, arrange for standing orders, and select individual titles or resources [11]. Libraries both physical and digital, usually have four common broad goals that help dictate these responsibilities. These goals are significant to libraries in order to maintain the basic principle of access.

1. To acquire material as quickly as possible.

2. To maintain a high level of accuracy in all work procedures.

3. To keep work processes simple, in order to achieve the lowest possible unit cost.

4. To develop close, friendly working relationships with other library units and vendors.

There are generally five steps taken in order to acquire material for a library collection whether physical or digital.

1. Request processing

2. Verification

3. Ordering

4. Reporting (fiscal management)

5. Receiving orders

There are eight different types of acquisition methods that are followed by libraries.

1. Firm orders - These are orders that are determined by name specifically. For example, a specific book, textbook, or journal that the library wants.

2. Standing orders - These are open orders for all titles that fit a particular category or subject. For example, these are usually developed for serials and the library knows that it will want anything published in that particular series. A benefit to this style of ordering is that it is automatic and the acquisitions department does not have to order the next in series.

3. Approval plans - These are normally similar to standing orders except they cover quite a few topic areas and are sent from the vendor and the library is only charged for the specific titles that they accept into their collection. Under these circumstances the library is free to return anything they do not wish to add to their collections. A benefit to this style of ordering is that the acquisitions department can sometimes make better decisions with the materials in hand versus an order form.

4. Blanket orders - These are largely a combination of both a firm order and an approval plan. Blanket orders are the library making a commitment to purchase all of something. For example, a library makes a contract with a certain publisher or vendor and will purchase everything that this publisher or vendor has available in regards to a topic. A benefit to this style of ordering is an automatic acquisition of materials for a particular field, which can be beneficial to specialized libraries or academic libraries.

5. Subscriptions - These are generally utilized for journals, newspapers, or other serials that a library will acquire. Like standing and blanket orders, a library only has to develop a contract once with a vendor or publisher and these are automatically delivered when they are printed. Many times subscriptions are for a period of time and must be renewed at the end of the contract.

6. Leases - These are contracts that allow access to particular resources for a period of time. Leases are most commonly utilized with electronic resources such as databases, journals, and web-based materials. The library is paying for access to the material versus paying for ownership of the material.

7. Gifts - In some cases libraries may allow gifts that people give to the library. It is the job of the acquisitions department to determine whether or not the gift will be kept and incorporated into the library's collection. This is most generally a method of large academic institutions and the persons giving the gifts are mostly alumni of that institution. It is stated within a collection development policy as to whether or not the library will accept gifts.

8. Exchanges - These can be broken into two subcategories: exchange of unwanted duplicate or gift materials AND the exchange of new materials between libraries. Again the institutions that usually have a process for this type of acquiring are larger academic or research libraries. This is also a process of consortia [5].

A Web service is a method of communication between two electronic devices over a network. It is a software function provided at a network address over the Web with the service always on as in the concept of utility computing. The W3C defines a Web service generally as a software system designed to support interoperable machine-to-machine interaction over a network [12].

\section{Related Work}

"Ontology-based Personalized Diet Plan Web Service Using HL7 Health Screening Data" The growth of medical technology extremely extends people's long of ages. A lot of people try to keep fit and firm, The healthy diet has become increasingly important today. This research describes the design and development of an ontology service (Diet-Aid) to generate personalized plan consistently based on personal information health. Diet interoperability issues in health and personal data by adopting the international standard HL7 as the input format. Moreover, Diet-Aid was developed and deployed using the RESTful architecture, allowing for anywhere any time any device to access through any Internet-enabled. And generate a personalized food advice on the spot. However, it would be done for increasing the nutrition about the admixture, such as amount of vitamins in the ingredients. Therefore, it could be enhanced by a 
matching process to the recommended daily intakes of nutrients. Another interesting aspect is to let the user view a diagram showing the indicator of nutrition balance to the meal chosen [4].

"Mobile application market: A developer's perspective" Major software companies, such as Apple and Google, are disturbing the relatively safe and established actors of the mobile application business. These newcomers have caused significant structural changes in the market by imposing and enforcing their own rules for the future of mobile application developments. This research, it described the implications that different market and technology trends have on the mobile application development market. The current evolutions show that the game has changed dramatically for developers. There are many new opportunities for them to develop, distribute, and generate significant revenues with the emerging mobile application portals [1].

"Software engineering issues for mobile application development" This research provides a snapshot of important software engineering research issues related to the development of applications that run on mobile devices. Among the topics are development processes, tools, user interface design, application portability, quality, and security. While application development for mobile devices goes back at least 10 years, there has been exponential growth in mobile application development. Since then, device makers have created outlets for other mobile devices, including Android, BlackBerry, Nokia Ovi, Windows Phone, and more. Industry analysts estimate that there are more than 250,000 applications available through the various stores and marketplaces, some of which are available for multiple types of devices [3].

"Applying Solr by Web Service for Modularized Learning Object Retrieval" Learning Object Retrieval Applications have been useful not only for providing users with the searching capability of educational contents, but also for exploring the degree of resemblance among subjects belonging to several courses of a certain educational institution. This prototype is shown on how the effective Web services of educational retrieves can be realized [8].

Therefore, this paper suggests to use web-service because it's provide convenient of communicate through mobile application, web-based application, database system and staff system on the international standard, market and technology trends. The mobile application has many applications available through the various stores and marketplaces.

\section{Methodology}

This research is a development research which offering library resources through web-site and mobile systems with web services to Central library Suratthani Rajabhat University and obtains assessment satisfaction from users. The representative sample and data for this research were compounded into 362 items, directed interview from 3 acquisition librarians, obtain data from staffs of 5 faculties included 73 from Faculty of Education, 92 from Faculty of Humanities and Social Sciences, 128 from Faculty of Science and Technology, 80 from Faculty of Management Science, 42 from Faculty of Nursing, 35 from Faculty Law, 68 from Faculty of International School of Tourism and 400 samples from the students of Suratthani Rajabhat University.

\subsection{System Architecture}

This is a system architecture for develop this system we used the web-service for share all data to each component.

The Fig. 1: The system architecture connected to the staff system of Suratthani Rajabhat University through web-service to import the staff data to this database. This system provides the web-service to web-based application and mobile application.

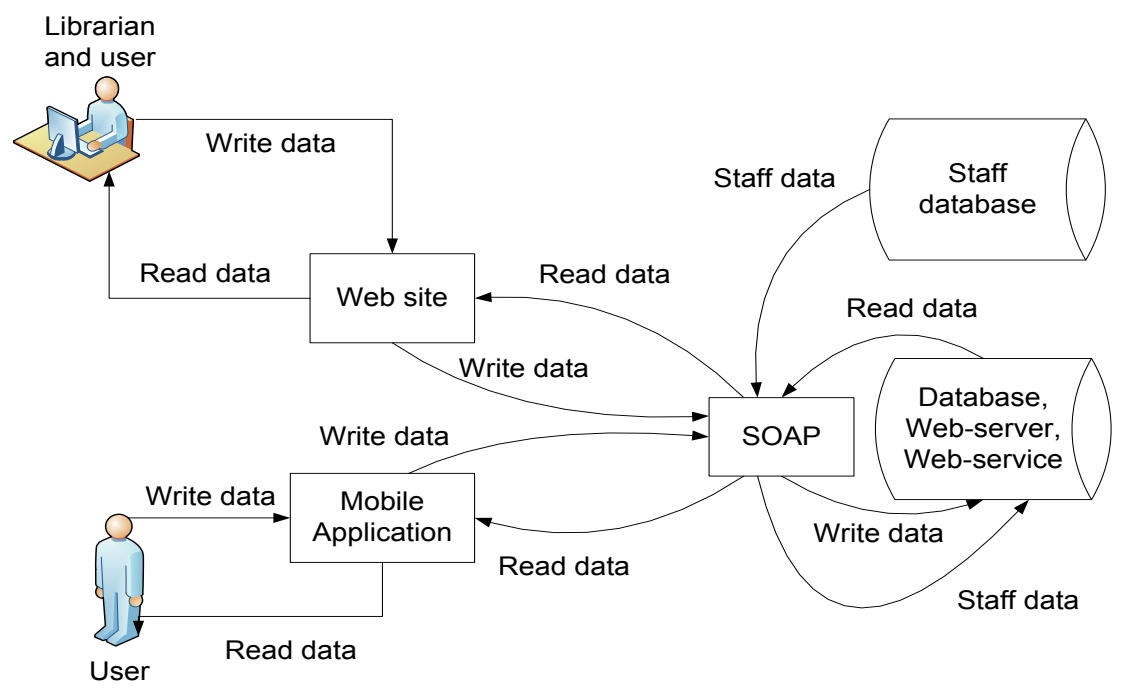

Figure 1. System architecture 


\subsection{The Method for Representative Sample}

The study is determined using Taro Yamane formula. This formula is used where the population parameter for the study is known. Thus it is stated:

$\mathrm{n}=\mathrm{N} / 1+\mathrm{N}(\mathrm{e}) 2$

Where: $\mathrm{n}=$ Sample size

$\mathrm{N}=$ Population size

$\mathrm{e}=$ Degree of tolerance error

With a confidence level of $95 \%$, the degree of tolerance error is $5 \%(0.05)$.

\subsection{Structure Chart}

This is a structure chart for develop this system. We show the breakdown of a system to its lowest manageable levels. They are used in structured programming to arrange program modules.

The Fig. 2: The Structure chart is consists of 8 components. They are member management, offering determine, offer and duplicate check, search, order, get items, keyword management and the last one is generate of report.

\subsection{Data Flow Diagram}

This is a Data Flow Diagram. It shows a graphical representation of the "flow" of data through an information system and shows what kind of information will be input to and output from the system, where the data will come from and go to, and where the data will be stored.

\subsubsection{Context Diagram}

The Fig. 3: The Context diagram of this system has 4 entities. They are: firstly, member entity. The inputs data of member entity are member data, offer data and key word. The outputs data are offering status, items data and member data. Secondly, web-service entity. The input data of web-service entity is call functions and the output is response data. Thirdly, librarian entity. The inputs data of librarian entity are member data, offer data, offering status, order, and key word. The outputs are offering status, items data and member data. And the last one of entity is staff system. The input data of staff system entity is member data. Another the output is requested of member data.

\subsection{Input and Output Design}

Input and output are the communication between an information processing system and the outside world, possibly a human or another information processing system. Inputs are the signals or data received by the system and outputs are the signals or data sent from it.

1) Home page design

This is a home page that is generally the first page a visitor navigating to a website from and may also serve as a landing page to attract the attention of visitors. The home page is used to facilitate navigation to other pages on the site.

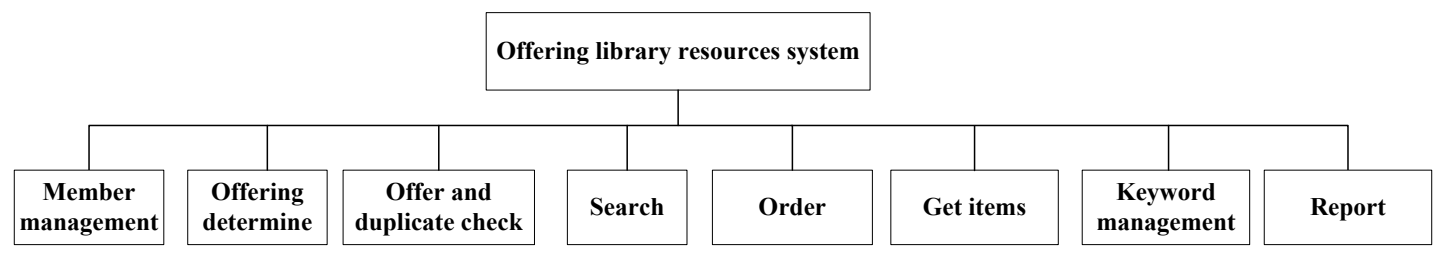

Figure 2. Structure Chart

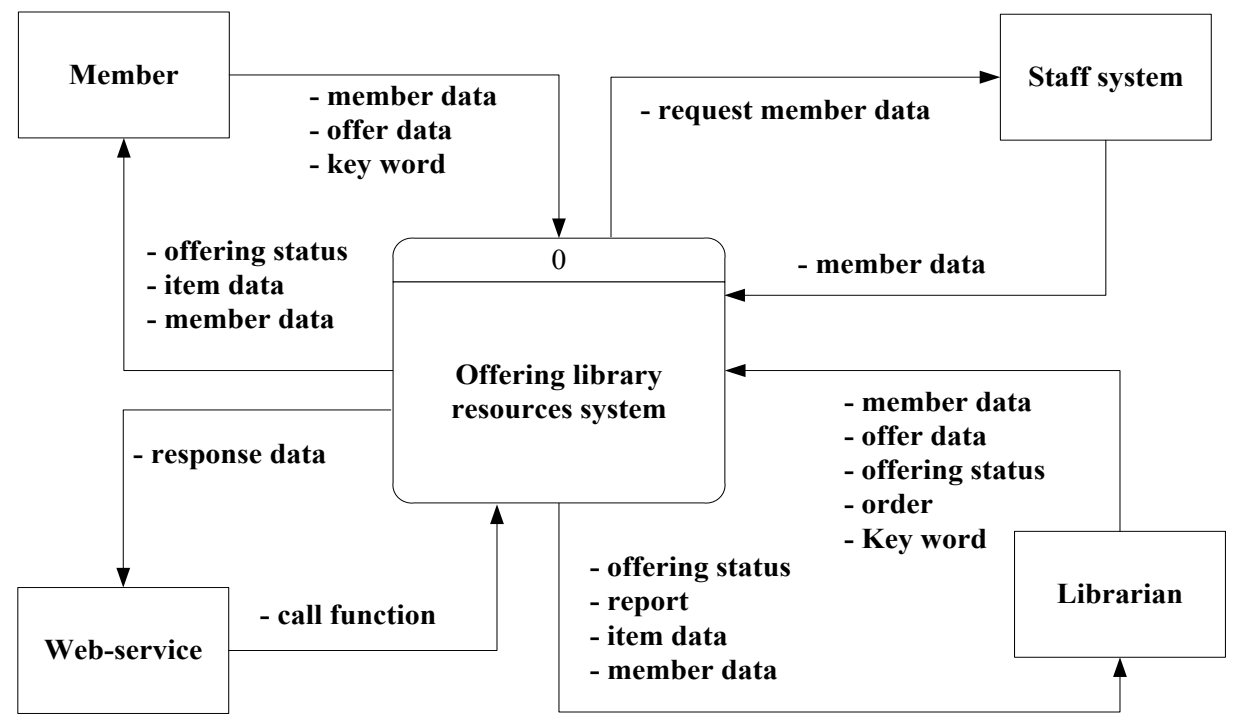

Figure 3. Context Diagram 
The Fig. 4: The home page design is composed of the header which the logo and system name. On the left side was the menu such as login, register and other. On the right side, the new items showed and the bottom is the footer. Which show organization information and the name of programmer.

\begin{tabular}{|c|c|}
\hline \multicolumn{2}{|c|}{ Logo and System name } \\
\hline $\begin{array}{c}\text { Menu } \\
\text { Login, Register and Other }\end{array}$ & New items \\
\hline \multicolumn{2}{|c|}{ Footer } \\
\hline
\end{tabular}

Figure 4. Home page design

\section{2) Input page design}

The Fig. 5: The offering page design on the web-site (on the left side) and the mobile application (on the right side) are compose of the title, author, year of publish, name of publisher, ISBN, offering amount, price, item type, description, request date and the last one is cover picture.

3) output page design

The Fig. 6: The cancel item offering report consists of the ID, title, author, member's offering, offer date and reason of cancel.
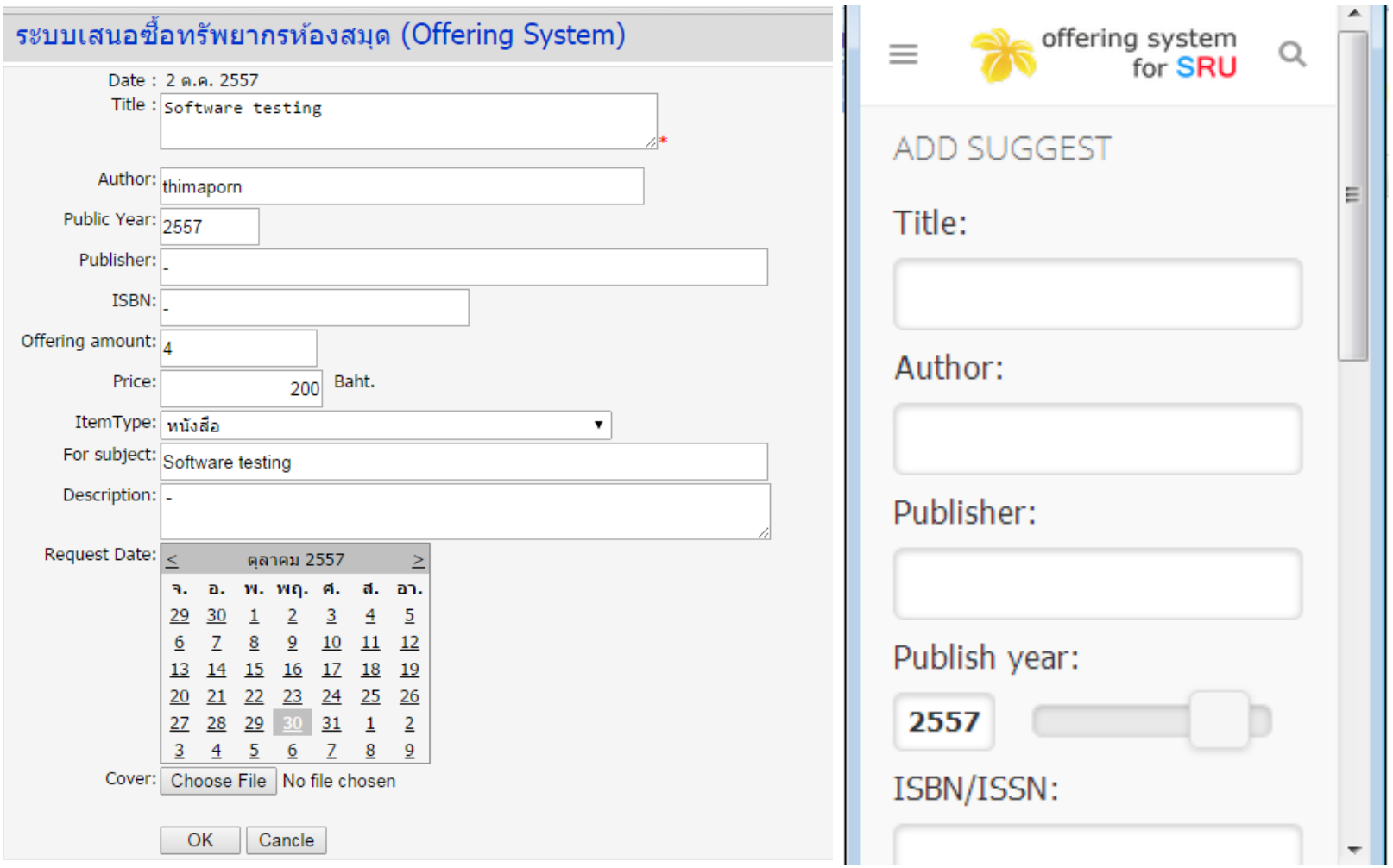

Figure 5. input design (Offering page design)

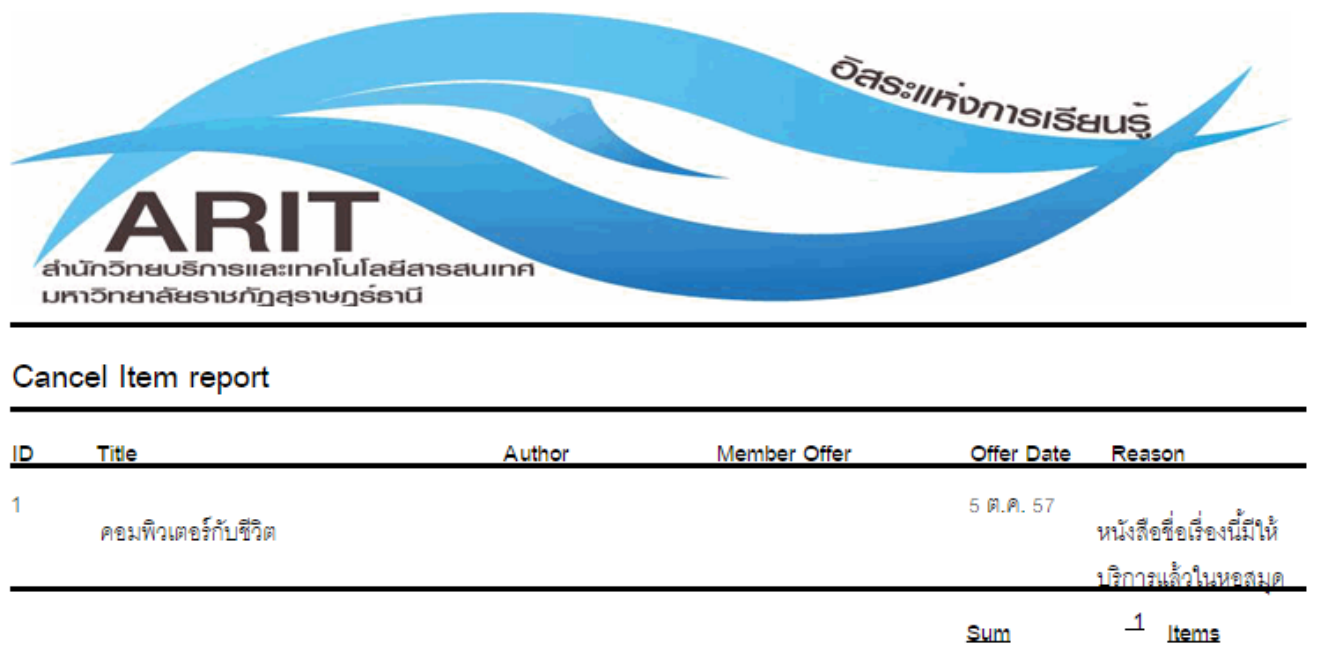

Figure 6. Cancel offering report 


\section{Implementation}

\subsection{The web-service was used for development of this} research. It was called from web-based application and mobile application

The Fig. 7: All of web-service functions. On the left side that's compose of insert, delete and edit faculty, department, item type, key word, member, member type, offer and offering history. And on the right side shows the example for calling and testing web-service functions. This function is insert member. The inputs of this function includes of member names, member surnames, faculty names, department names, telephone numbers, email address, username, password and member type of ID.

\subsection{Those are the home pages after we installed the system}

The Fig. 8: The home pages of this system on the web-site (on the left side) and the mobile application (on the right side).

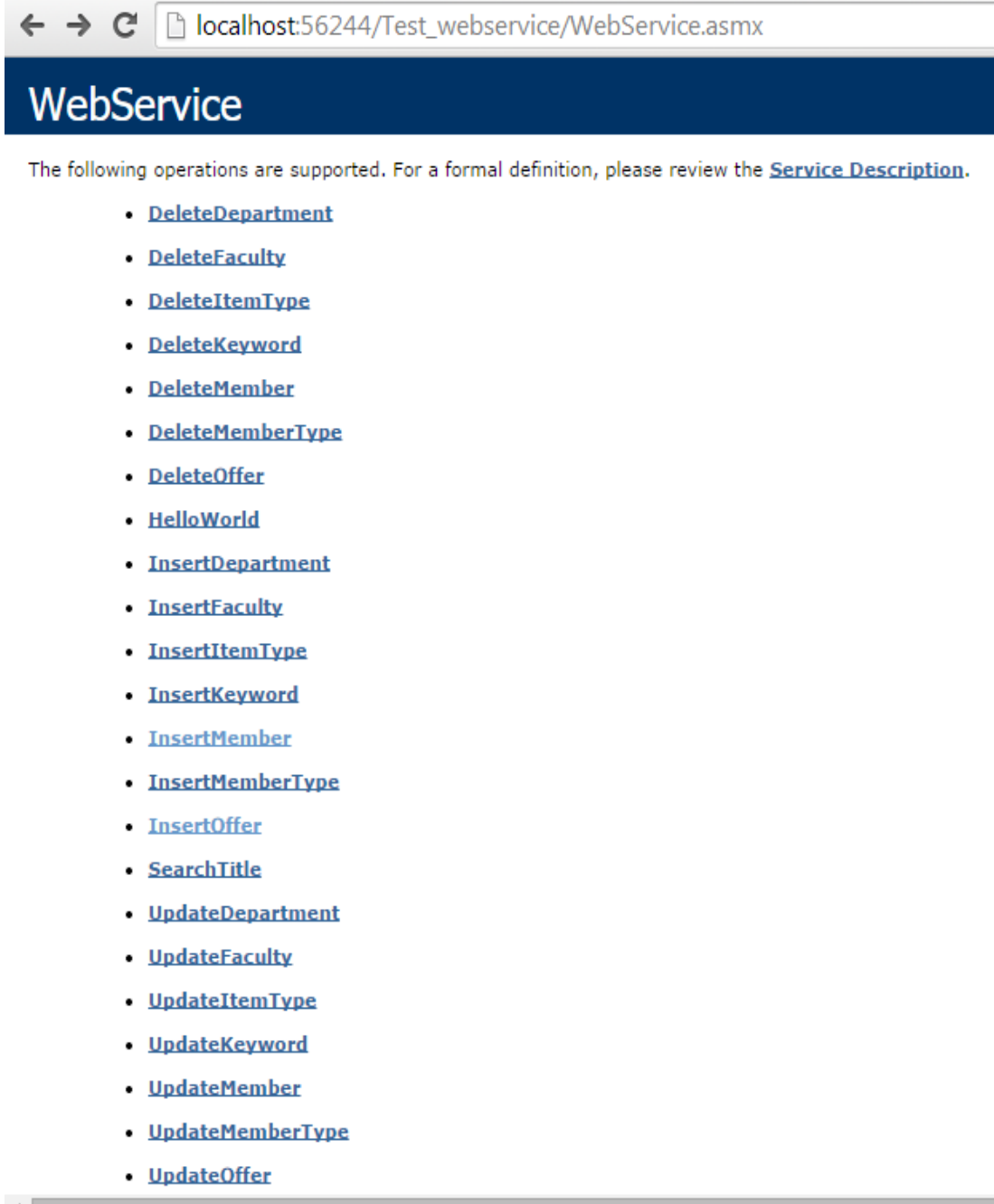


$\leftarrow \rightarrow$ C $\square$ localhost:56244/Test_webservice/WebService.asmx?op=InsertN

\section{WebService}

Click here for a complete list of operations.

\section{InsertMember}

\section{Test}

To test the operation using the HTTP POST protocol, click the 'Invoke' button. Parameter Value

GetMemberName:

GetMemberSurname:

GetFacultyID:

GetDepartmentID:

GetMemTel:

GetMemEmail:

GetUsername:

GetPassword:

GetMemberTypeID:

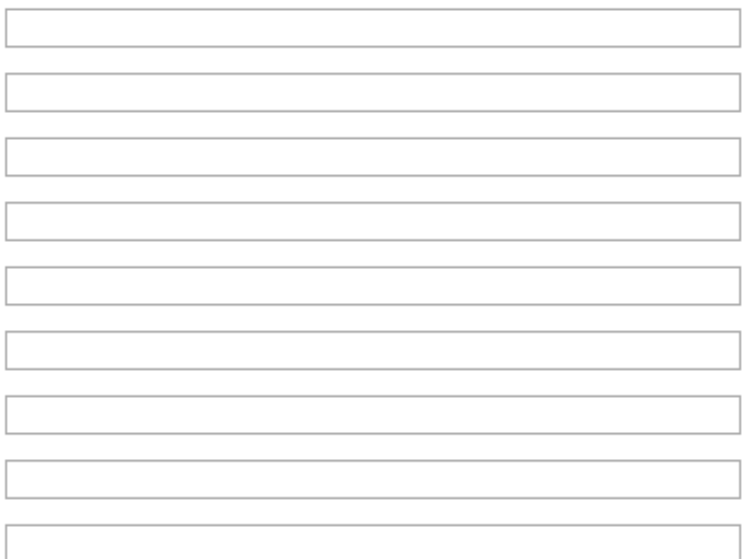

Invoke

\section{SOAP 1.1}

The following is a sample SOAP 1.1 request and response. The placeholders shown need to be pOST / Test webservice/WebService.asmx HTIP/1.1 Host: locainost

Content-Iype: text $/ \mathrm{xml}$; charset=utf-s

Content-Iype: text $/ \mathrm{xm}$;

Content-Iength: length
gokpaction: "http://tempuri.org/InsertMember"

$\langle ? \mathrm{xml}$ version="1.0" encoding="ut $f-8 " ?\rangle$

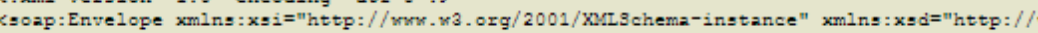
4

Figure 7. Show all web-service functions

\section{C [9 localhost:49246/Offering_system_2_10_57/index.aspx}

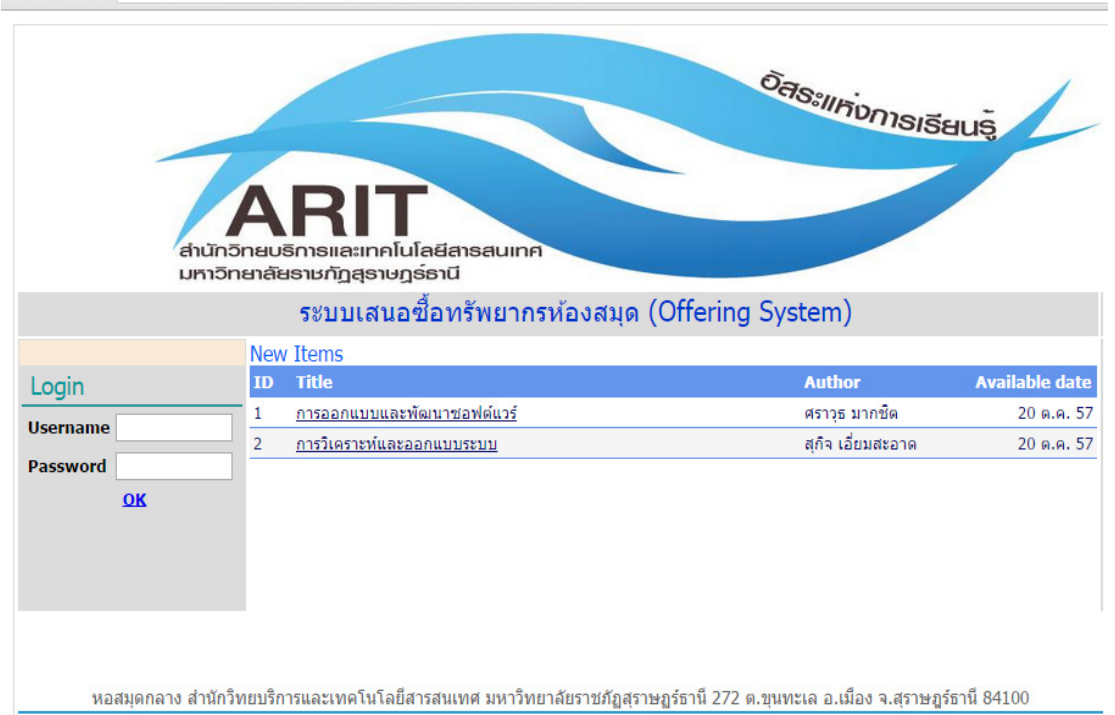




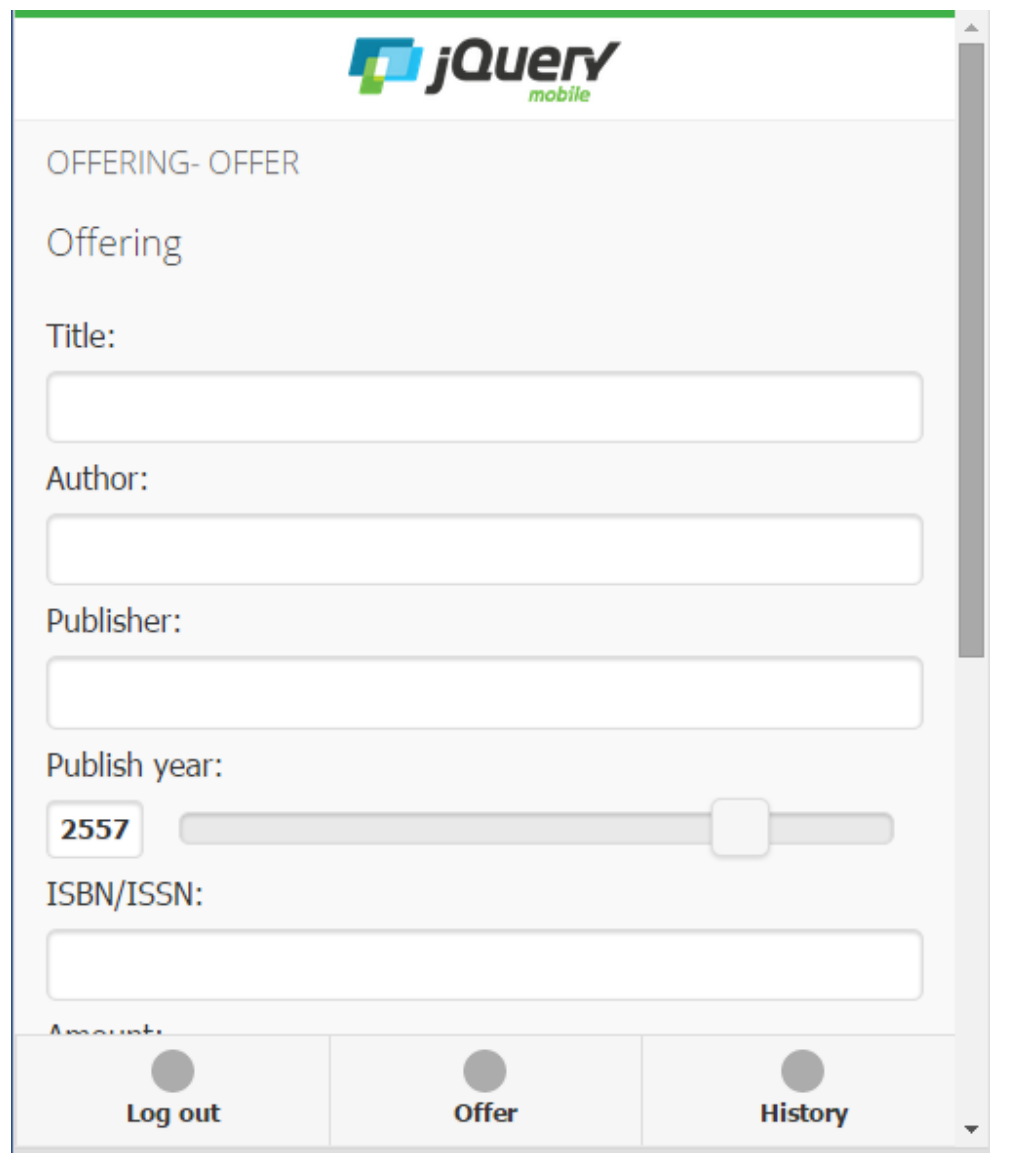

Figure 8. Show the home page

\section{Conclusions}

This paper aim to develop the web-service serve to other components because it's convenient of communicate through the mobile application, web-based application, database system and staff system as tantamount in the international standard, market and technology trends. The results of this were analyzed, designed and developed the system. After installed the system, assess for the users satisfaction such as easily to use, method of output, prompt service, accuracy, security, completely, increase efficacy for work and benefit from the system showed that the total average as satisfactory. The researcher suggestion to improve the connect of library system to check the duplicate title in the further.

\section{REFERENCES}

[1] Adrian Holzer, Jan Ondrus, Mobile application market:A developer's perspective. Telematics and Informatics, pp. 23-31, 2011.

[2] Allen, R. E., ed. (1984). The Pocket Oxford Dictionary of Current English. Oxford: Clarendon Press: p. 421.

[3] Anthony I. Wasserman, Software engineering issues for mobile application development. Proceedings of the FSE/SDP workshop on Future of software engineering research, pp. 397-400, 2010.
[4] Chuan-Jim, Chia-Wen Chih and Yin-An Chen, Ontilogy-based Personalized Diet Plan Web Service Using HL7 Health Screening Data. Proceedings of the Asia Pacific Industrial Engineering \& Management Systems Conference, pp. 1164-1175, 2012.

[5] Evans, G. and Margaret Zarnosky Saponaro. 2012. Collection Management Basics: Sixth Edition. Libraries and Information Science Text Series. Libraries Unlimited; Oxford, England.

[6] Liz Chapman. 2001. Managing acquisitions in library and information services. London: Library Association Publishing.

[7] Peter Clayton and G.E. Gorman.2001. Managing information Resources in libraries. London: Library Association Publishing.

[8] Teerasak pengmusaw and sunton witosurapot, Applying Solr by Web Service for Modularized Learning Object Retrieval. Proceedings of The 6 TH National Conference on Computing and Information Technology, 2010.

[9] Yamanae Taro.1973. Statistics: An Introductory Analysis. London: John Weather Hill, Inc.

[10] Library-Definition and More from the Merriam-webster Dictionary. Meriam-webster.com. Retrieved 23 March 2015.

[11] Reitz, Joan M. acquisitions. Online Dictionary for Library Information Science. ABC-CLIO. Retrieved 23 March 2015.

[12] Web service Glossary. W3C. Retrieved 23 March 2015. 